Extrême-Orient Extrême-Occident

\section{Extrême-Orient Extrême-Occident}

39 | 2015

Corps souffrants dans les littératures de la Chine et du Japon au XXe siècle

\title{
Le corps souffrant dans la littérature chinoise depuis la Nouvelle période (1979-2015)
}

Suffering Bodies in Chinese Literature Since the Reform and Opening (1979-2015) 徐爽，阿麗亞娜·德·奧麗維拉·格麥斯。簡析新時期以來中國文學中的 痛楚身體(1979-2015)

Xu Shuang et Ariadna de Oliveira Gomes

\section{(2) OpenEdition} Journals

Édition électronique

URL : http://journals.openedition.org/extremeorient/532

DOI : $10.4000 /$ extremeorient.532

ISBN : 978-2-84-292-449-2

ISSN : 2108-7105

\section{Éditeur}

Presses universitaires de Vincennes

\section{Édition imprimée}

Date de publication : 1 octobre 2015

Pagination : 145-176

ISBN : 978--84292-447-8

ISSN : 0754-5010

Référence électronique

Xu Shuang et Ariadna de Oliveira Gomes, «Le corps souffrant dans la littérature chinoise depuis la Nouvelle période (1979-2015) », Extrême-Orient Extrême-Occident [En ligne], 39 | 2015, mis en ligne le 01 octobre 2017, consulté le 20 avril 2019. URL : http://journals.openedition.org/extremeorient/532 ; DOI : 10.4000/extremeorient.532 


\title{
Le corps souffrant dans la littérature chinoise depuis la Nouvelle période (1979-2015)
}

\author{
Xu Shuang et Ariadna de Oliveira Gomes
}

La première moitié du $\mathrm{XX}^{\mathrm{e}}$ siècle est en Chine traversée par les guerres et les révolutions. C'est pourtant la fin de ce siècle et singulièrement les années de l'essor économique qui voient apparaître, sous la plume des écrivains, l'image récurrente d'un corps affaibli, torturé ou malade : récurrence qui interroge. Tout se passe comme si le corps ne pouvait être détaché du thème de la douleur ; la mise en scène du corps souffrant est figure iconique et figure rhétorique, image valant par elle-même et symbole renvoyant à un champ de parole difficile, voire impossible. Ce qui ne peut ou ne sait se dire s'inscrit, et s'inscrit somatiquement. Le corps se grave, comme une plaque d'imprimerie, et souffre cette inscription avant même, peut-être, de savoir la lire.

La scène de l'expression somatique est singulièrement complexe. En amont du texte littéraire, le discours officiel donne l'exemple : il n'est pas indifférent que, dans la propagande et particulièrement l'exercice très chinois du slogan politique, le corps ait servi et continue à servir de métaphore privilégiée. Dans les années 2000 encore, le but proposé par les autorités à la nation se dit xiaokang - la «prospérité modeste »-terme dont les éléments impliquent la bonne forme physique (kang, santé).

À cet égard, la manipulation du discours pendant la période maoïste a constitué à la fois un modèle et un extrême. La transformation que proposait le régime pouvait être décrite par Mao en une image brutalement somatique : il s'agit, pour l'homme modelé par la révolution, de "se métamorphoser jusqu'aux os et faire peau neuve »(tuotai huangu) ${ }^{1}$. Mieux encore, la définition du corps se déplace ; le corps, qui signifiait l'individu, doit devenir un élément d'uniformité. Au début des années 1960, Lei Feng (1940-1962), soldat légendaire des contes éducatifs de la propagande maoïste, exprime le nouvel idéal en des termes aussi violents que significatifs : «La fonction d'un

1. Voir Mao $1963: 430$. 
individu, par rapport à la cause révolutionnaire, est comme celle d'une vis dans une machine. [...] Je veux être une vis pour toujours ${ }^{2}$.» Le seul corps considéré devient ainsi celui de la nation révolutionnaire, qui n'est plus un organisme, mais une "machine », à l'intérieur de laquelle l'être humain ne possède pas l'identité minimale d'une fonction, mais est un rouage interchangeable, une « vis » qu'on peut forger - modelage par la gymnastique imposée sur le lieu de travail - ou refondre pour un nouveau façonnage - « rééducation » par les travaux agricoles ou les travaux forcés (laogai).

Il n'est pas moins significatif qu'au lendemain de cette fusion en une grande unité révolutionnaire, le détachement des hommes rejetés au rivage de l'individualité trouve un mot expressif : shanghen, la " cicatrice ». Shanghen est à la fois le titre d'une nouvelle de Lu Xinhua (1954-) et le terme de ralliement d'un mouvement littéraire qui doit son nom à celle-ci. La « littérature des cicatrices » a pour fonction, selon Lu Xinhua, de faire état de la «gigantesque cicatrice que dix années de troubles ont laissée sur le peuple chinois $^{3} »$. Le texte littéraire dénonce ainsi la métaphore mise en place par le discours politique : la nation n'est pas le gigantesque mécanisme que la propagande a voulu voir en elle, elle est faite d'une chair et d'un sang pour lesquels le façonnage programmé par le régime a été une chirurgie à vif dont le résultat reste opaque : la « cicatrice » signifie-t-elle une guérison, certes lourde de mémoire, mais acquise, ou au contraire, la trace d'une amputation irréversible?

Pour intéressante que soit cette question, qui soutiendra notre lecture de la littérature des quelque trente-six années qui nous séparent de l'instauration de la politique dite de Réforme et d'Ouverture qui a succédé à la Révolution culturelle, ce n'est pas elle que notre analyse se proposera comme essentielle. Il existe en effet un acquis de la Révolution maö̈ste dont il importe de savoir s'il doit être considéré comme durable et s'il conditionne la vision du corps dans la littérature contemporaine : si la nation souffre des séquelles d'un traumatisme physique, d'une « cicatrice », ce n'est pas seulement parce que ses membres ont vécu une tragique expérience commune, c'est parce que cette expérience interroge le rapport de l'individu au groupe. Qui est, finalement, corps ? Est-ce l'homme ? Est-ce le «peuple chinois » ? Et quelle est la souffrance du corps ? Celle de s'inclure dans un grand ensemble dans lequel il peut tout au plus prétendre à une fonction, ou bien de s'en voir détaché ?

La question ainsi posée permet d'interroger le paradoxe que nous allons parcourir au fil de la chronologie. La « cicatrice », le corps souffrant sont

2. C'est un extrait du Journal intime de Lei Feng, voir Li 2009.

3. Zhongguo wenxuejia cidian $1985: 110$. 
des représentations liées au souvenir traumatique des années difficiles, mais ils ne s'arrêtent pas à celles-ci : le thème de la douleur physique héritée de la Révolution culturelle se retrouve en littérature, en d'autres termes et sous d'autres formes, dans la représentation des maux issus de la consommation et des dégâts collatéraux de la croissance économique. Le corps apparaît ainsi dans l'univers littéraire comme un objet dépourvu d'indépendance, voire comme le principe d'une aliénation qui s'exprime dans une interrogation souvent brutale sur l'inhumain, le sexe et l'amour, la violence et la mort.

\section{Corps docile et corps meurtri}

Au commencement était le corps docile : tel pourrait être le résumé de la question du corps, telle qu'elle apparaît dans le souvenir immédiat des lendemains de la Révolution culturelle. Par corps « docile», il faut entendre, à la suite de Foucault, un corps « qu'on manipule, qu'on façonne, qu'on dresse, qui obéit, qui répond ${ }^{4} »$. Surveiller et punir note que « dans toute société, le corps est pris à l'intérieur de pouvoirs très serrés, qui lui imposent des contraintes, des interdits ou des obligations $»^{5}$, alors que les corps vivants sont par nature « plaisir, discontinuité, fête, repos, besoin, instants, hasard, violence, etc. ${ }^{6} \gg$. Parler d'un corps docile, c'est donc interroger l'accès au désir et, par là, l'accès au plaisir.

\section{$L$ ' « étranger de retour » : corps amer}

De quelle manière le corps de la jouissance et de la fête, du hasard et du besoin devient-il donc « corps docile »? C'est ce qu'interroge un des textes importants de la « littérature des cicatrices », la nouvelle Un étranger de retour (Guilai de moshengren $)^{7}$ de Bei Dao. L'auteur y relate le retour au foyer d'un intellectuel réhabilité au lendemain de la Révolution culturelle. Victime d'une fausse accusation, jugé « coupable de crimes contre le peuple », l'homme a passé vingt ans en camp de travail pour rééducation.

4. Foucault $2014: 160$.

5. Ibid. : 161.

6. Harcourt (dir.) $2013: 236$.

7. Bei Dao (1949-, né à Pékin) est un poète et un romancier qui s'est fait connaître dès les années 1980. Cette nouvelle a d'abord été publiée dans la revue non officielle Jintian 今天 (Aujourd'hui), puis dans le recueil éponyme Un étranger de retour (voir Bei Dao 1986). Nos citations en français se réfèrent à l'édition française (voir Bei Dao 1999). 
Le récit est donné à la première personne, avec pour narratrice la fille du héros, restée séparée de son père depuis l'âge de cinq ans. Dans le regard de celle-ci, l'homme apparaît dès son arrivée comme un individu privé d'autonomie, impuissant à maîtriser le cours de son existence. Tel « un marin roulé par la vague », " il a lutté avec l'énergie du désespoir pendant cette longue vie d'errance, jusqu'à ce qu'une autre vague, comme par miracle, le rejette sur le pont d'origine ${ }^{8} »$. Cette « longue vie » ne fait l'objet d'aucun discours mais s'inscrit dans le signifiant somatique : le corps du père est déformé, affaibli et passif ; sa face est « blême », " couverte de cicatrices », ses mains apparaissent « raides », avec des « articulations déformées par l'arthrite ». La voix elle-même s'est transformée, « basse », tremblante ; son ton est de manière permanente « compatissant et implorant ${ }^{9}$ ».

La dégradation physique se traduit pour la narratrice en un contenu narratif : le corps est une surface qui s'offre au décryptage. La perte de «dignité » du père est perçue comme l'écho d'une humiliation que la fille reconstitue (fantasme ?) comme engendrée par la menace des « barbelés », des « mitrailleuses » et de « la mort ». Ainsi, les barbelés ne se résument pas à l'existence concrète d'un fil de fer, pas plus que le camp n'est un périmètre défini dans un espace réel : ils constituent des univers mentaux dont la victime ne peut se libérer. Même lorsque, de retour chez lui, il est « hors d'atteinte », libéré de la présence du « chef de brigade », éloigné du camp de travail, le père ressent de manière physique la menace du regard d'autrui. Ainsi, ayant jeté un paquet de cigarettes sans vérification, le front en sueur, il se lève à l'aube et se précipite pour aller fouiller la poubelle. La réalité historique vécue, reconstituée à partir de la trace physique et des gestes du père, est cependant aussitôt retranscrite par la narratrice dans les termes du corps : l'homme mentalement détruit lui paraît physiquement «pétri » «comme de la glaise».

Bien que le récit se termine par le rétablissement progressif du lien affectif entre père et fille, le corps du rescapé reste figé comme un témoin du passé. La posture mutique, "soumise ", du père s'impose comme une trace et un symbole : «Il serre fortement les lèvres, comme s'il avait avalé sa langue ${ }^{10}$. » Elle illustre la manière dont s'est imposé le «carcan » de l'appareil politique, terme qu'il ne faut pas percevoir comme une métaphore lexicalisée, mais avec la dimension concrète que suppose le supplice hérité de l'empire et ressuscité par la Révolution culturelle : «[...] nos maîtres d'école embellissaient la

8. Ibid.: 19 .

9. Ibid.: 28 .

10. Ibid.: 21 . 
vie, l'entouraient d'un nimbe. [...] En fait de nimbe il s'agissait plutôt [...] d'anneaux de fer servant de carcan ${ }^{11}$ !»

Le corps prostré ne met pas en cause la seule existence d'un sujet livré aux vicissitudes de l'histoire. Il se fait contagieux. En retrouvant un père sans voix, la narratrice voit se ranimer en elle le souvenir de la «blessure » ancienne, qu'elle croyait effacée :

«Mon père ?» ai-je murmuré pour moi-même, et je me suis détournée brusquement, comme terrorisée par le sens de ce mot. Puis mon cœur s'est mis à battre la chamade. C'était clair : les fils qui suturaient l'ancienne blessure avaient lâché ${ }^{12}$.

Il existe ainsi une différence essentielle entre le signifiant de «père » et ce même mot donné en présence du référent réel. Les violences subies par la narratrice comme fille de condamné reviennent à sa mémoire à mesure qu'elle perçoit les traces laissées par ces violences sur le corps-miroir du père. La souffrance individuelle, de spectacle qu'elle était, se transforme en expérience partagée. C'est au terme de ce partage que le lien se recompose, et que la famille trouve moyen de retrouver existence - peut à nouveau faire corps. Car c'est bien là le sujet du roman : si le corps d'un homme rendu « docile » ne peut retourner à la vie ordinaire, un autre corps est à trouver ou retrouver. Finalement, la grande souffrance provoquée par la Révolution culturelle n'aura pas été de briser l'homme mais de séparer, de disjoindre les membres d'un organisme d'un autre type, familial, et d'empêcher le père de voir grandir sa fille tout en contraignant la fille à grandir sans père.

\section{Corps frustré : «La moitié de l'homme, c'est la femme »}

Cette peinture de la douleur physique due à la disjonction des corps plus encore qu'à la souffrance individuelle se dit d'une manière plurielle. Dans le roman La moitié de l'homme, c'est la femme (Nanren de yiban shi nüren) ${ }^{13}$, de Zhang Xianliang (1936-2014), l'expression de cette souffrance est simple et crue, c'est-à-dire sexuelle. Le corps commun disjoint se présente ici comme un « demi-corps » pour lequel le contact avec sa «moitié » (la femme) est rendu impossible : le lien impraticable de la pénétration sexuelle invalide la constitution de l'unité de vie familiale.

11. Ibid.

12. Bei Dao $1999: 21$.

13. Voir Zhang 2009 pour l'édition chinoise et Zhang 2004 pour l'édition française. Nos citations se réfèrent à l'édition chinoise. 
La moitié de l'homme, c'est la femme se présente sous les auspices du fantasme de l'impuissance sexuelle. Le protagoniste, l'intellectuel Zhang Yonglin, est la victime des mouvements politiques qui se sont succédés depuis la campagne anti-droitière de 1957. Il a passé dix ans de sa jeunesse entre camps de rééducation et prisons. Libéré à 39 ans, il retrouve la femme dont il est secrètement amoureux, la sensuelle Huang Xiangjiu, qu'il avait aperçue huit ans auparavant dans le camp de travail, alors qu'elle se baignait nue dans une rivière. Il l'épouse, elle l'aime. Mais la nuit de noces, face au corps tant désiré de sa femme, Zhang Yonglin s'avère incapable de consommer l'acte sexuel. Humilié par son handicap, le héros laisse le chef de la commune nouer une liaison avec son épouse.

Le sujet était, pour un roman paru en 1985, hardi : le succès de Zhang Xianliang a été d'abord lié au parfum de scandale que laissait planer, en ces débuts de la Nouvelle Période, la mise en scène de la nudité et du coït ${ }^{14}$. Soumis à la morale puritaine de l'époque maoïste, le protagoniste se présente dès le départ comme associant la question sexuelle avec des représentations effrayantes : il tient le désir pour «un démon » (mozhang) - terme issu du canon bouddhique ${ }^{15}$-, dont la présence est « capable de détruire la sagesse, la morale, la culture et la vertu ${ }^{16} »$. La sensation que le corps éprouve sous l'effet de l'appétit sexuel est rendue par un ensemble d'images très négatives. Agressive et blessante comme « un objet pointu et déstabilisant qui attaque au bas ventre », elle est « sournoise » (yinxian), immorale, destructrice, littéralement monstrueuse :

(Le désir) fondrait très probablement sur moi, me déchirerait en morceaux avant de lécher ses lèvres ensanglantées et de se jeter sur la première femme qu'il apercevrait ${ }^{17}$.

En surprenant le corps nu de la femme, l'homme éprouve une tension inhibitrice qui fait naître en lui le sentiment d'un affrontement à une catastrophe ; il tremble devant ce qui lui apparaît comme un piège (xianjin), un effondrement $(\text { duoluo })^{18}$. Cette torture, à la fois physique et spirituelle, résulte du jeu de

14. Voir Li 2014.

15. Le mot est tiré du Traité de la grande vertu de sagesse de Nāgārjuna (Dazhidu lun 大 智度論) : ouvrage du III ${ }^{\mathrm{e}}$ siècle, traduit en chinois par Kumârajîva (344-413). Pour la traduction en français, voir Lamotte (trad.) 1980.

16. Zhang $2009: 142$.

17. Ibid.: 142 .

18. Ibid.: 142-143. 
deux forces en conflit : «d'une part la réflexion rationnelle qui se fonde sur la foi en une croyance, liée à la contrainte et à la surveillance exercées par une civilisation ; d'autre part, l'instinct irrationnel, l'aspiration à s'unir à un corps charnel vivant, réel, à ce corps de femme que je vois de mes yeux et qui stimule mon désir ${ }^{19}{ }$. Cette inhibition se traduit dans les faits : jusqu'à son mariage à l'âge de trente-neuf ans, le héros n'a jamais touché réellement le corps d'une femme ${ }^{20}$, bien que celui de Huang Xiangjiu ait été, des années durant, l'objet de fantasmes aussi violents que réprimés.

En contrepoint, la féminité de Huang Xiangjiu, l'épouse, resplendit. Elle apparaît d'abord nue lors d'une baignade secrète. Se sentant observée à la dérobée derrière les roseaux qui s'agitent doucement au vent, elle " ne se presse pas de se rhabiller; lâchant la culotte qu'elle tient à la main, elle croise les bras devant sa poitrine comme si elle avait froid et tourne son corps vers moi [le protagoniste] ${ }^{21} \gg$. La chair s'exhibe ainsi au regard masculin :

Sans aucun geste de séduction, sans un mot, sans un sourire, elle m'appelle par ses regards, par le frisson de chaque parcelle de sa peau, par l'innocence de sa présence $^{22}$.

La nature du féminin se révèle dans le détail des gestes : quand Huang Xiangjiu enlève la paille qui colle à son habit, jette un coup d'œil au miroir ou range la maison... À une époque où les deux sexes sont appelés à s'aligner sous une apparence asexuée et uniforme ${ }^{23}$, la féminité menace l'homme et la séduction apparaît sous les couleurs de la fatalité. L'appel mis en avant par le corps de Huang Xiangjiu se révèle ainsi en contradiction, ou plus exactement en concurrence avec l'ambition politique du régime. Aux yeux de l'appareil, l'individu standardisé par le port de l'uniforme s'identifie à sa « force de travail » (laodongli) qui l'intègre au grand corps de la nation; tandis que le sexe suppose un autre type de fusion :

La grâce de ce corps nu suscite en l'homme que je suis un désir, une passion. Il me fait comprendre ceci : bien que je sois « une force de travail», je n'en suis pas

19. Ibid. : 171 .

20. Ibid. : 142-143.

21. Ibid.: 153-154.

22. Ibid. : 154.

23. Voir Roberts 2009. 
moins, sous la surface noire, bleue ou verte, un homme. Dans cette uniformité qui gomme l'individualité, il me reste au moins mon $\operatorname{sexe}^{24}$.

La contradiction se met à jour dans l'opposition entre la jouissance éprouvée dans le désir et la souffrance que provoque la rencontre réelle avec le corps désiré. L'incapacité sexuelle est rendue par des métaphores violentes, dans lesquelles se connotent le danger, la mort, le dégoûtant : marais, volcan, mollusques céphalopodes avec tentacules à ventouses directement rattachés à leur tête, éponge aspirante, mirage du désert ${ }^{25} \ldots$ La perte de l'accès au corps par la sexualité amène ainsi le sujet au diagnostic identitaire de la disparition, en la personne de sa femme, d'une partie de son corps :

Je ne suis qu'une « moitié d'homme » (ban ge ren), « un estropié » (feiren $)^{26}$.

Dans un second mouvement dialectique, cependant, l'impuissance sexuelle se fait métaphore politique. Le protagoniste, intellectuel soucieux de l'avenir du pays, établit un lien d'analogie entre la passion politique et l'appétit charnel :

L'excitation politique ressemble à la pulsion du désir. Toutes deux proviennent d'une sécrétion du corps (nei fenmi) qui incite l'homme à pénétrer courageusement, à avancer, posséder, se procurer satisfaction et joie dans l'action ${ }^{27}$.

Pour le héros, perdre sa virilité équivaut ainsi à perdre sa capacité à intervenir dans l'espace collectif. Un dialogue fantastique avec le cheval du village souligne l'écart qui sépare le corps mutilé du grand historien Sima Qian, victime du supplice de la castration sous l'empire des $\operatorname{Han}^{28}$, et le moderne corps impuissant du protagoniste. Tandis que celui-là trouve dans la réaction à la violence éprouvée la force d'achever ses Annales historiques (Shiji), celuici, en proie à un « mal » qui « a pénétré dans les viscères, dans la tête, dans les nerf ${ }^{29}$ », est réduit à l'inaction. En cela il subit à la fois la position de la femme dans l'acte sexuel et celle de la bête domestiquée (du cheval) :

24. Zhang 2009: 171.

25. Ibid.: 201.

26. Ibid. : 209, 226-227.

27. Ibid. : 178.

28. Sima Qian 司馬遷 (vers 145-vers 86 avant J.-C), historien chinois.

29. Zhang $2009: 208$. 
Le corps souffrant dans la littérature chinoise depuis la Nouvelle période

Tu laisses les autres te manipuler, te fouetter, te monter dessus ${ }^{30}$.

À la fin du discours, le cheval fantastique persifle l'intelligentsia humiliée en trouvant encore une fois le registre du corps : "Je me demande si tous ces intellectuels n'ont pas été castrés », déclare l'animal.

Le rôle joué par le corps du protagoniste est, comme on le voit, pensé selon deux logiques. D'une part, il apparaît comme le moyen qui permet au sujet d'accéder à une libération de la contrainte collective : par la sexualité, le personnage peut enfin revivre le moment d'intimité initié des années plus tôt dans le secret de la rivière ; le couple lui fournit la communauté restreinte, minimale, qui doit lui permettre de survivre à la grande aliénation collective. Mais, d'autre part, ce corps reste pensé comme l'outil de la relation entre individu et groupe, homme singulier et ensemble social : sans l'élan viril de la pulsion, l'homme ne saurait accéder à une fonction réelle, celle-ci se réduisîtelle à celle de l'intellectuel qui, sur l'exemple de Sima Qian, loue ou blâme le fait du prince - les agissement du chef de la commune avec Huang Xiangjiu par exemple, que le héros subit sans pouvoir les condamner. Dans les deux cas, c'est par le corps et singulièrement par la fonction virile de celui-ci que le sujet comprend son accès au(x) groupe(s) ; l'impuissance interdit aussi bien l'intimité conjugale que le travail collectif. Le dénouement du roman est à cet égard significatif : le protagoniste se débarrasse de son impuissance lorsqu'il se montre capable d'une action héroïque lors d'une crue. Parallèlement, le dégel politique se profile avec l'annonce radiophonique de la prise de pouvoir de Deng Xiaoping, qui laisse naître, chez les intellectuels contemporains, la possibilité d'un nouvel engagement.

La frustration du protagoniste sert de support à une analyse au terme de laquelle le corps joue entre une représentation asociale parce qu'asexuée et une sexualité devenue acte politique. Au terme d'une fable transparente, on voit l'intervention politique maö̈ste interdire, puis confisquer la vie intime du personnage tandis que la Réforme et l'Ouverture autorisent à nouveau sexualité conjugale et utilité sociale. Loin d'imposer un lavage de cerveau, ou encore, comme dans les littératures de la Shoah, de donner en spectacle les extrêmes de la violence irrationnelle, l'oppression est un phénomène organique, dont l'action essentielle se déroule dans le secret des corps. Faute d'échapper aux techniques de sujétion, l'individu devenu physiquement docile est en souffrance aussi bien au plan biologique (sexualité) qu'intellectuel (aspiration politique).

30. Ibid. : 208 . 


\section{«Qui suis-je ? » : corps halluciné et « génies malfaisants »}

C'est à la liaison de la réflexion sur articulation entre le monde physique et l'univers psychologique que la nouvelle de l'auteure Zong Pu (1928-) "Qui suis-je ? (Wo shi shui) ${ }^{31}$ offre l'un des meilleurs récits consacrés au questionnement identitaire, poursuivi à travers la représentation des corps aliénés. «Qui suis-je ?» relate le destin d'un couple de scientifiques qui adhère volontairement au régime communiste et le paie par d'atroces souffrances.

Après leurs études à l'étranger, Weimi et son mari choisissent de rentrer en Chine au printemps 1949 par exaltation patriotique et par admiration pour la Révolution, qu'ils voient comme un «creuset glorieux ». Ils décident de «faire peau neuve » et de « se forger dans le haut fourneau de la Révolution ${ }^{32}$ ». Cependant, au début de la Révolution culturelle, ils sont condamnés comme « droitiers », « traîtres », « ennemis du peuple », et soumis à des tortures physiques et mentales. Ils subissent les violences et les humiliations des séances de critique publique : le crâne à demi rasé, ils sont frappés et fouettés ${ }^{33}$. Ils restent cependant fidèles au régime, acceptant la punition et se résignent à balayer le campus «en silence, mécaniquement ». Pourtant, après avoir vu brûler le fruit de ses travaux scientifiques, le mari choisit de soulager ses souffrances en faisant usage de la seule liberté qui lui reste et se pend dans la cuisine. Weimi, poursuivie par les accusations politiques, altère progressivement l'interrogation identitaire en un délire hallucinatoire ; des corps fantastiques et des génies malfaisants lui apparaissent. De terrifiants épisodes délirants alternent avec d'autres où, lucide, l'héroïne s'interroge sur son propre état : «Est-ce que les serpents savent rire ? Ce n'est que dans le monde de la folie que de telles choses peuvent se produire ${ }^{34}$.» Parallèlement, son comportement public manifeste la démence qui la mine : elle crie « J'ai tué quelqu'un! » ou hurle « Je suis vénéneuse, surtout ne me touche pas $^{35}$ ! ». Elle acquiert la certitude qu'elle empoisonne la jeunesse et que son savoir scientifique propage un venin fatal. Le lecteur est témoin du déchirement qui

31. La nouvelle a été publiée dans la revue Changchun 長春 (décembre1979); nos citations se réfèrent à la version française, voir Zong 1994. Il est intéressant de l'associer à une autre nouvelle à la même thématique et du même style : « Ma coquille » («Woju »), parue dans la revue Zhongshan 鍾山 (janvier 1981), voir Zong 1988.

32. Zong $1994: 37$.

33. Ibid.: 33 .

34. Zong $1994: 36$.

35. Ibid.: 35 . 
l'envahit et la pousse finalement, dans un destin parallèle à celui de son mari, à la noyade.

Il faut donner ici toute son importance à la focalisation interne poursuivie au long du récit : tandis que, pour les personnages qui constituent son entourage, l'état de la narratrice se manifeste comme un phénomène mental (elle est « la folle »), le récit homodiégétique met en scène un vaste espace fantastique dont le héros et l'enjeu se confondent avec le somatisme protéen de la femme délirante. «Le corps est absent », dit Foucault dans Le Pouvoir psychiatrique $^{36}$ : le corps docile, qui souhaitait se muer en un être neuf, se nie en des formes inédites. Weimi se voit ainsi comme un « diable bovin, un serpent démoniaque » (niugui sheshen), un démon " au visage terrifiant, à la cruauté infinie ». " De ses mains démesurément grandes elle poursuivait une foule variée de petits enfants tous joliment parés ${ }^{37} »$; le thème onirique des paumes immenses qui battent les enfants, hérité de Lu Xun, prend ici l'épaisseur concrète de l'hallucination ; la persécutée harcèle, puis empoisonne et tue : sous les huées qui l'invectivent, elle se mue en une « fleur blanche » qui diffuse de dangereuses « lueurs phosphorescentes ${ }^{38}$ », avant de se transformer en « reptile» (chongzi), en « gros serpent venimeux » (da duchong $)^{39}$. La métamorphose physique se réinterprète aussitôt comme identification du sujet à son persécuteur : les démons qui fournissent leur forme à Weimi lui apparaissent comme l'irruption animalisée des tortionnaires dont elle a été la victime ; devant le regard terrifié de la protagoniste, une petite colline prend la forme de « visages de démons hideux », les cavités d'un roc aux lueurs du couchant deviennent « autant de bouches béantes et sanglantes ${ }^{40} » \ldots$ Nous ne sommes plus ici, comme chez Bei Dao, dans la description de la dégradation physique, ou, comme chez Zhang Xianliang, dans la dimension symbolique de celle-ci, mais dans un pied-de-la-lettre psychotique et poétique à la fois, au terme duquel le mot se fait chose. Les injures - niugui sheshen, da duchong, outils privilégiés de la persécution politique - deviennent autant de corps, diables, figures animales et démoniaques. Le procédé atteint son apogée dans les dernières lignes de la nouvelle, où le signe écrit apparait comme une annonce certaine de l'avenir avant de révéler son ambivalence sémiotique. Sur le point de se noyer, Weimi voit les oiseaux dessiner dans le ciel « une forme brillante qui figure le caractère 人 (ren, homme) » et y lit l'espoir d'un monde

36. Foucault (dir.) et Grange $2003: 268$.

37. Zong $1994: 32$.

38. Ibid. : $34-35$.

39. Ibid. : 35-36.

40. Ibid.: 32 . 
enfin humain. Mais, à l'instant d'expirer, elle ne se perçoit plus que comme entourée de formes dégradées de l'humanité : des squelettes et des reptiles métaphoriques, dont elle a subi les attaques tout en perdant elle-même son humanité par identification à ces formes.

Le « corps absent » foucaldien prend ici tout son sens. Si Weimi est privée de rapports avec l'altérité au point d'en perdre la raison, c'est qu'elle a été dépossédée de l'identité physique minimale qui lui permettrait de reconnaître ceux qui l'entourent comme des semblables et d'être reconnue d'eux comme un être humain. Dans la nouvelle, les êtres extérieurs et l'héroïne n'assument les formes reptiliennes et démoniaques que dans des moments alternés ; quoi qu'il arrive, monstre ou femme, Weimi n'appartient dans son délire jamais à la même espèce que ceux qu'elle rencontre. La persécution aboutit ainsi à un double déficit : d'une part, l'héroïne n'est plus elle-même (identique à soi), d'autre part, elle n'est plus homomorphe à ceux qui l'entourent (identique aux autres). Le " corps absent », plus qu'une absence de corps, est surabondance d'identités somatiques et impossibilité d'un corps semblable, d'un corps commun. Il est manque parce qu'il est perte du corps des autres. Quoi qu'il arrive, et c'est bien là le destin de la victime, quand bien même elle fait partie de millions d'autres, elle est seule de son espèce, abandonnée à une souffrance isolée.

La « littérature des cicatrices » peut être perçue comme la forme du deuil « officiel » dédié aux tourments vécus durant la Révolution culturelle. Les premiers corps souffrants dans cette représentation littéraire sont essentiellement ceux des intellectuels, des cadres embourgeoisés et de leurs familles, victimes des violences politiques du régime. À partir de 1985, dans les courants littéraires qui s'affirment, le corps souffrant prend les formes les plus diverses : le corps déformé d'enfants infirmes comme le personnage de Bingzai dans Papapa de Han Shaogong ${ }^{41}$, le corps malade chez Zhang Wei dans Le Vieux bateau (Guchuan) ${ }^{42}$, la vie humaine représentée comme souffrance historique chez Yu Hua ${ }^{43}$, le corps livré à l'abjection, la monstruosité et l'animalité chez Mo Yan ${ }^{44}$, etc.

41. Voir Han 2000 pour l'édition française du roman et de Oliveira Gomes 2012 pour l'étude à ce sujet.

42. Voir Zhang 2014 pour l'édition française du roman. Pour l'étude du corps malade, voir Xu 2010 et Xu 2012.

43. Voir Rabut 2005.

44. Zhang 2014, pour l'étude de la représentation du corps dans l'ensemble de l'œuvre de Mo Yan. Voir Xu 2013a et Xu 2014, pour une étude du corps fantastique et celle du corps irrégulier chez Mo Yan. 
En fait, le temps du deuil est vite remplacé par « l'idéologie du développement économique ». Sur la scène littéraire apparaissent de nouveaux personnages, liés à l'essor de la société de consommation ${ }^{45}$. La question du corps se pose dans un monde ébloui par l'argent et la prospérité matérielle. Le traumatisme de la Révolution culturelle semble s'éloigner. Pourtant, l'analyse révèle que la question du corps, loin de disparaître dans un âge d'abondance, se présente sous des formes qui entretiennent avec la période précédente une proximité troublante.

\section{Deuil des âmes souffrantes et société de consommation}

En 1999, le poète Michel Deguy lance dans son adresse aux poètes français : «La poésie faisait mal ; fit mal ; savait faire mal. Pourrait-elle le faire encore ${ }^{46}$ ? ॥ne telle interrogation se retrouve chez le poète chinois Luo Ying (1956-) qui, dans son recueil Lapins, lapins (Xiao tuzi) ${ }^{47}$, surprend le lecteur par la vision d'un « enfer contemporain : urbain, climatisé, et peuplé d'inquiétants rongeurs. Décapant univers dans la lignée de Cioran ${ }^{48} »$. Luo Ying est un poète atypique dont le parcours est le témoin de l'histoire de la Chine depuis les années 1950, en particulier de celle qui a suivi la Révolution culturelle. Dans son œuvre, la présence du corps est la pierre de touche par quoi se déstabilise l'image d'une nation absorbée dans les seuls soucis de la prospérité économique.

\section{Corps aliénés ou la souffrance en temps d'abondance}

Lapins, lapins est composé de dix poèmes en prose écrits en 2006. La fureur, l'impuissance, la douleur, la critique sociale sont autant de thèmes qui en parcourent l'écriture. La réalité, caractérisée par les ténèbres, la cruauté et la débauche, relègue le locus amoenus de la poésie chinoise traditionnelle ; cela se marque dans un choix de couleurs dans lequel le rouge sang efface les teintes pastel ; les éléments de la lyrique classique - barque, nuage, nuit, pluie, aube, instrument à cordes, lune, étoiles, brume, crépuscule -, présents dans les précédentes œuvres du poète, s'absentent, détruits ou resémantisés :

\footnotetext{
45. Voir Xu 2011b.

46. Deguy 1999.

47. Luo Ying 2013.

48. Voir Jeancourt Galignani 2013.
} 
Ainsi, je vois mourir, les uns après les autres : montagnes, fleuves, villes entières. Je vois aussi les hommes mourir et tomber les uns après les autres, comme les cerises d'un arbre.

Et la terreur se répand et s'en nourrit. (« De la terreur $»^{49}$ )

Sans montagne ni fleuve, le narrateur lyrique ne peut plus prendre de barque pour voyager au lointain ou monter sur une hauteur pour se retirer du monde. Le poète se met en scène comme un esclave de son environnement stérile, apocalyptique, « saisi par la terreur au $\mathrm{XXI}^{\mathrm{e}}$ siècle ${ }^{50}$ ».

La civilisation industrielle détruit la beauté de la nature et de la douceur humaine. Ponctuant cette dégradation, Luo Ying retrouve l'image de la métamorphose apparue chez Zong Pu. Les corps prennent des formes animales : chiens errants, rats, cafards, corbeaux, serpents, vers dans les fosses d'aisance, mante religieuse qui tue son mâle, et enfin ces lapins éponymes, véritables allégories du corps sans force - pour survivre, ces créatures se laissent humilier jusqu'à l'abdication de toute dignité ${ }^{1}$.

Le poète écrit : "Il suffit de très peu de souffrance, d'une quantité infime, ou plus précisément d'un tout petit peu de mal émanant de l'esprit pour que nous-mêmes et notre existence tendions vers le sublime, et qu'au moins nous ne ressemblions pas à des vers grouillant dans une charogne, convives d'un écœurant festin » (《La souffrance $»^{52}$ ). Le corps humain est ici métaphoriquement évoqué par celui de l'invertébré élémentaire ; l'espace commun apparaît comme un gigantesque repas dans lequel ces créatures s'acharnent à dévorer « une charogne ». Dans cette allégorisation de l'individu ou plutôt des masses « grouillant[es] » affrontées au grand banquet d'une consommation dans laquelle la jouissance immédiate semble devenue un mot d'ordre, le poète met en scène une inversion des valeurs : « très peu de souffrance » ou « plus précisément» «un tout petit peu de mal» devient la condition sine qua non de l'accès au « sublime ». Cette souffrance doit se comprendre comme physique (il s'agit, concrètement, de ne pas avoir accès à la ripaille) mais aussi et surtout comme morale (puisqu'on peut, au nom de la souffrance, refuser cette ripaille). De ce phénomène complexe, la description est d'une concrétude organique qui recherche le dégoût : la mise à mal de la spiritualité dans la société de consommation devient « un écœurant festin » dans lequel le plat proposé au public est entré en décomposition

49. Luo Ying $2013: 25$.

50. Ibid.

51. Ibid. : 71-6.

52. Ibid.: 37 . 
depuis longtemps. Les laissés-pour-compte du développement dans une société d'abondance apparaissent ainsi comme d'étranges élus que la douleur empêche de toucher à un mets certes plantureux, mais dangereux. Dans un paradoxe du même type, le poète représente l'homme en lapin " asexué » qui, malgré l'absence d'organes, « doit se masturber en public, mimer à de nombreuses reprises les positions de l'acte sexuel, prenant la place du mâle ou de la femelle ${ }^{53} »$; la métaphore réédite l'aventure contradictoire mise en scène par Zhang Xianliang : l'incapacité d'accès au repas commun se transforme en une sexualité sans issue, pour laquelle aucune position, tant physique que sociale, n'est possible, si ce n'est celle, solipsiste, de la «souffrance».

Dans La Neuvième Nuit (Di jiu ye, 2008) ${ }^{54}$, achevée deux ans après Lapins, lapins, l'interrogation sur le corps se creuse d'un retour sur la question de l'animalité. Le recueil se compose de deux grandes parties : "Le cheval - expérience sexuelle d'un cheval et son impasse morale » et « Le chat - nuit de défloration d'une chatte et récit extraordinaire de sa mort ». Le critique Yang Xiaobin voit dans cette évocation de la sexualité des animaux domestiques l'image de la jouissance sexuelle telle qu'un corps métamorphosé l'appréhende tant en intensité qu'en vitesse ${ }^{55}$.

À l'aide de mon phallus qui traverse les siècles et les époques, je soulève le gong du gardien de nuit pour appeler les disparus ${ }^{56}$.

Le son du « gong » dans la nuit suggère le réveil des endormis, et plus métaphoriquement la société obscure d'où sont absents les acteurs, les héros. Le désir d'éveil s'exprime à travers l'image du phallus en érection, symbole de « jouissance » qui domine le recueil. Cependant, dans « Le cheval », cette « jouissance » contemporaine révèle sa signification ambiguë. Source de volupté, elle s'avère létale, puisqu'il s'agit pour le personnage de se tuer au terme de neuf nuits de plaisir :

Se tuer soi-même au bout de neuf nuits est la décision douloureuse d'un mutant monstrueux ${ }^{57}$.

53. Luo Ying $2013: 75$.

54. Luo Ying 2011.

55. Ibid. : 4 .

56. Ibid.: 38 .

57. Ibid.: 94. 
Le cheval, métaphore transparente d'une sexualité de la performance, devient « le spécimen d'un symptôme et d'une mutation » non moins lisibles. Ce qui n'empêche que le corps éprouve la frustration du désir, car la jouissance tant recherchée s'avère, au bout du compte, insatisfaisante. Dans la course au plaisir en effet, l'organe sexuel, bien qu'omniprésent, est représenté comme invalide ou insignifiant. Il est découpé, congelé, inepte et finalement détaché du corps. La représentation de la castration revient au terme du texte, comme l'expression d'une fatalité ; la jouissance est décrite comme vécue sous une menace imminente :

Dans une époque barbare comme ce $\mathrm{XXI}^{\mathrm{e}}$ siècle, un phallus traverse les missiles, les fusils ; il échappe à la guerre en Irak, il s'efforce, avant la castration, de faire jaillir la dernière goutte de son sperme ${ }^{58} \ldots$

Dans le recueil Tara $(L \ddot{u} m u d u, 2009)^{59}$, la représentation du corps souffrant parce qu'isolé reprend l'anthologie des thématiques que nous avons repérées. Le locuteur « je » se voit tour à tour «prisonnier » (qiutu) ${ }^{60}$ et «esclave » $(\text { nuli })^{61}$. Victime de la violence extérieure, il en porte, comme le personnage de Bei Dao, les stigmates physiques : yeux aveugles, dents brisées, os fracturés, orteils pulvérisés, pieds amputés, voix étouffée ${ }^{62}$. Son enfermement ne s'interrompt que dans les métamorphoses ; celles de Zong Pu sont empruntées à un appareil fantastique démoniaque, celles du protagoniste de Tara ont une forme animale : papillon, pigeon, serpent, corbeau, cerf, cheval, poisson, singe, cheval, crapaud, grenouille ${ }^{63}$. La perte de la capacité physique illustrée chez Zhang Xianliang devient ici une auto-mutilation, mise en image dans des scènes où l'individu « se dévore », « boit son propre sang », " se fait mal », «se coupe les pieds », « se croque les jambes », ou « se découpe en tranches de chair ${ }^{64}$. Le corps subit un anéantissement d'autant plus violent qu'il est partiel : «j'erre avec, à la main, la viande hachée tirée de mon corps »; la chair entre en lente putréfaction sur l'individu vivant ${ }^{65}$. La décomposition spirituelle

58. Ibid.: 54-55.

59. Luo Ying 2013.

60. Ibid.: 157.

61. Ibid. : 205, 238 .

62. Ibid. : 204,$231 ; 204 ; 214 ; 215 ; 215 ; 220$.

63. Ibid. : 3,$190 ; 191 ; 193 ; 193 ; 193 ; 209 ; 230,239 ; 239 ; 238 ; 239 ; 245$.

64. Ibid. : 209, $241 ; 210 ; 212 ; 240 ; 222 ; 222$.

65. Ibid. : $222 ; 195$. 
parachève cet ensemble; des chenilles « déchirent (silie) tout » et l'âme « se débat (zhengzha) » dans le bec d'un corbeau ${ }^{66}$.

Les trois recueils, Lapins, lapins (2006), La Neuvième Nuit (2008) et Tara (2009), qui proposent une image du corps aliéné par la consommation, la recherche contemporaine de la performance, la violence autodestructrice de l'homme isolé, retrouvent les thématiques allégoriques de la " littérature des cicatrices »; la représentation obsessionnelle des « cicatrices » aux mains et au visage ( Qui ai-je tué67 ${ }^{6}$ ) constitue une convergence troublante. Au seuil du nouveau siècle, dans un univers social stable et prospère, le poète se voit condamné à la souffrance physique totale que le demi-siècle précédent avait comprise comme la rançon expiatoire de la pénurie et des mécomptes politiques :

Je ressemble à un oiseau mort, accroché à l'arbre et exposé au regard de tous Je suis la proie de cette époque couverte de traces de balles

(« Le soleil se lève $\left.(2)^{68} »\right)$

Faut-il pour autant comprendre la « littérature des cicatrices » comme un paradigme d'engendrement à partir duquel il faille envisager la totalité de la production post-maoïste, de 1979 à aujourd'hui ? La textualité serait-elle, dans une contemporanéité au sein de laquelle les jeunes générations chinoises ignorent parfois jusqu'au nom de la Révolution culturelle, figée dans un traumatisme?

\section{Corps souffrants et mémoire : diagnostiquer le mal}

Il est certain que cette dernière interrogation n'est pas absente de l'œuvre de Luo Ying. En 2012, le poète achève un long texte autobiographique consacré au souvenir de la Révolution culturelle et aux suites de celles-ci, Le Gène du garde rouge (Wenge jiyi) ${ }^{69}$. Il s'agit pour l'auteur de « purger un passé qui hante non seulement un homme incapable de trouver le sommeil mais la nation chinoise tout entière ${ }^{70} »$.

Le recueil est composé de quatre parties. Les deux premiers chapitres sont consacrés aux souffrances de l'époque maoïste. Les deux derniers traitent de la

66. Ibid. : $157 ; 184$.

67. Ibid. : 125 .

68. Ibid. : 123 .

69. Luo Ying 2015.

70. de Clercq $2015: 227$. 
période actuelle. Parlant depuis le XXI ${ }^{\mathrm{e}}$ siècle, le poète affirme sa résolution de « rouvrir la plaie du passé », et de décrire souffrances et violences collectives ; il le fait sous la forme de condoléances, dans le contexte, hérité de la tradition bouddhique, d'un deuil manifesté pour apaiser les morts inassouvis. Par-delà la dénonciation des responsables ou l'expression de la douleur qui avaient fait le fond de la « littérature des cicatrices », le poète cherche, par cet acte pieux, à « libérer l'âme » des corps restés en souffrance. Ces derniers sont ainsi mis en scène dans une tentative de diagnostic général.

\section{La souffrance : coupable et victime}

Dans une première scène, le père du narrateur, accusé d'être un « contrerévolutionnaire », est « ligoté » et «emmené » par les « révolutionnaires ». Le détail physique passe par la description de la grosse corde de chanvre rêche qui enserre ses bras et sa gorge :

Cette corde devait le blesser au cœur ; il ferma bien les yeux

Puis baissa docilement la tête, en bon soldat discipliné

Colonel de l'Armée de campagne, il avait dû tuer de nombreux soldats du Parti nationaliste

(«Mon père, tas d'os desséchés $\left.{ }^{71} »\right)$

Le père est victime d'une injustice politique, accablé par la maltraitance physique et morale. Mais, sous un autre angle, il apparait comme un des auteurs des massacres qui ont accompagné la précédente révolution, dans laquelle il était « colonel de l'Armée de campagne ». Le personnage qui accompagne sans révolte ses bourreaux est en réalité un ancien rouage de l'appareil politique :

Père avait combattu lorsque la révolution avait eu besoin de combattants

S'était sacrifié lorsque la révolution avait exigé le sacrifice ${ }^{72}$

Ainsi, ancien «colonel » et actuel « contre-révolutionnaire », le père se laisse traîner « tête basse, comme un chien », sans réaction, « en signe de loyauté » ${ }^{73}$. Militaire, il ne bronche pas contre la violence de la révolution en laquelle il conserve sa foi.

71. Luo Ying 2015 : 16.

72. Ibid.: 18.

73. Ibid. : 17-18. 
Il en va de même pour le narrateur. Fils du contre-révolutionnaire, il fait l'objet d'humiliations sociales, souffre de l'injustice politique, mais, comme écolier et petit garde rouge, n'en participe pas moins à la lutte contre les « mauvais éléments » et devient l'auteur indirect de la mort d'un propriétaire foncier et du directeur de son école ${ }^{74}$.

La violence s'étend à l'ensemble d'un corps social mis en accusation ambiguë. Contre-révolutionnaires fusillés, propriétaires fonciers torturés à mort, ou « garde rouge tombé sous le couperet ${ }^{75}$ » : la révolution s'achève en un massacre général.

\section{Corps collectif et sacralisation du pouvoir}

Un des points les plus intéressants du recueil consiste en la description précise du façonnement physique auquel se livre l'appareil politique. Le révolutionnaire est pourvu par l'exercice gymnique d'un mouvement normé, uniforme et collectif. «La danse de la loyauté » illustre le façonnage d'un corps qui s'inclut, au terme du processus, dans l'unité supérieure concrète que constitue la collectivité, tant politique («nous l'exécutions lors des défilés, des débats ») que familiale (« ainsi qu'à la maison») :

La danse de la loyauté visait à porter la révolution au plus profond de l'âme

Nous l'exécutions lors des défilés, des débats, ainsi qu'à la maison

Poings serrés, tête levée, il fallait bomber le torse en gardant les yeux écarquillés, fixés au loin

Elle m'évoque à présent la posture du Diamant, celle du gardien du temple de Bouddha

(« La danse de la loyauté » ${ }^{76}$ )

Comme toute danse, celle-ci exige une maîtrise des gestes. Chaque mouvement doit être minutieusement décomposé et défini. Les corps adoptent conjointement des attitudes figées, respectent avec précision la manière de se tenir, de marcher, d'exécuter les ordres, de travailler. Le mouvement sacralisé est finalement intégré au rituel de la vénération du pouvoir ; celui-ci n'a ainsi plus qu'un interlocuteur (un adorateur) en la personne du corps unique dans

74. Voir «Le petit garde rouge du président Mao» (ibid. : 41) ; « La lutte contre les propriétaires fonciers » (ibid. : 55).

75. « Le garde rouge tombé sous le couperet », Luo Ying $2015: 79$.

76. Ibid.: 45 . 
lequel se sont fondues les «poupées politiques ${ }^{77}$ » individuelles. Cette unité bouge, s'immobilise ou articule d'une manière unanime :

Nous devions crier : «Vive le président Mao ! » et rester immobiles, figés dans une chorégraphie collective

Nous portions l'uniforme militaire jaune, ceinturon à la taille, insigne de Mao étincelant sur la poitrine ${ }^{78}$

Le désir lui-même s'exprime sous forme globalisée et l'hymne entonné par tous résonne aussi bien en chacun; la voix même lie indissociablement l'individu et le groupe :

La danse de la loyauté était la façon qu'avait mon âme d'exprimer le désir de révolution

Chaque jour à l'aube résonnait en moi l'hymne solennel : «L'Orient est rouge ${ }^{79}$ Tourné vers l'horizon, je serrais fort le poing

Tandis que montait en moi l'envie féroce d'anéantir le monde entier ${ }^{80}$

Dans ce processus, « le petit livre rouge », lecture unique et communautaire, devient un ouvrage sacré, dont la couleur « rouge et or éclatant » qui « s'illuminait de rayons dorés ${ }^{81} »$ symbolise l'accord moral qui correspond à la pratique de la voix et du geste collectifs. Il en va de même pour un cadeau offert par le président Mao en personne - « une mangue couleur d'or », qui « brillait d'un éclat inouï » et dont le parfum « se répandait dans la ville entière ${ }^{82}$ ».

Dans le poème «La mangue », le texte décrit d'abord l'enthousiasme qui accueille l'objet sacré, véritable moment de folie collective : la ville «passa trois jours à l'attendre sans fermer l'œil / Les gens tapaient sur des gongs et des tambours, exécutaient la danse de la loyauté ; les rues s'étaient vidées de dix milles personnes venues l'accueillir le long de l'artère principale / le visage baigné de larmes, tous criaient à tue-tête : Vive le président $\mathrm{MaO}^{83} »$. Le fruit divin se transforme cependant rapidement en une source de conflit. Les

77. Foucault $2014: 161$.

78. Luo Ying $2015: 45-46$.

79. Hymne de la République de Chine pendant la révolution culturelle. Le président Mao y est comparé au soleil levant et qualifié de sauveur du peuple.

80. Luo Ying $2015: 45-46$.

81. Ibid.: 43 .

82. Voir « La Mangue », Luo Ying $2015: 47$.

83. Ibid. 
différentes factions révolutionnaires se disputent la possession de la mangue et la rue passe de la transe collective à une lutte brutale, qui ramène sur le devant de la scène les dissensions particulières jusque là niées ; l'hystérie joyeuse se transforme en un " champ de bataille résonnant de coups de feu ${ }^{84}$ » et la fête s'achève sur la vision de cadavres accumulés. La scène a révélé la tragédie du corps collectif : malgré l'emprise de la répétition litanique, celui-ci se découvre destiné à l'éclatement de l'existence particulière, véritable « catastrophe » dont il est la « victime $(\text { sinanzhe })^{85}$.

\section{Le corps souffrant comme objet de jouissance}

Déclaré révolutionnaire ou jugé criminel, l'individu qui se détache de la masse est désigné à la violence : l'exposition des condamnés à la foule (shizhong) est une représentation récurrente dans le recueil. Dans cette position, l'être humain est ramené à l'existence la plus élémentaire : sur la pancarte qu'il promène, son nom dit son individualité, le chef d'accusation indique sa particularité, c'està-dire son crime (droitier, contre-révolutionnaire, etc.) ; cet ensemble le réduit bientôt à l'état de corps anonymé, torturé, violenté publiquement, exécuté.

Le peuple se rue vers la scène : "Les frères Wu furent jetés dans un camion, exhibés dans les rues pour servir d'exemple / [...] On les mena à la mort. La ville entière était en effervescence, comme si une fête battait son plein ${ }^{86}$. » Lors de la lutte entre des mineurs révolutionnaires et des gardes rouges, " toute la ville assistait au combat, sans boire ni manger ; les gens applaudissaient, criaient Hourra ! Hourra ${ }^{87}$ ! $»$. Au début du XX ${ }^{\mathrm{e}}$ siècle, Lu Xun (1881-1936) avait déjà noté l'attitude des hommes du peuple qui contemplaient avec « un détachement tout esthétique » le spectacle de " l'exposition à la foule ». Sébastien Veg note dans son étude consacrée à l'auteur que « cette jouissance esthétique » est placée chez lui « sous le signe du théâtre »; dans la nouvelle «La véritable histoire d'A-Q », l'épisode qui précède l'exécution est «fortement théâtralisé »; on revêt le condamné d'un costume, le promène en ville dans une charrette et le spectacle s'achève sous « les bravos de la foule ${ }^{88}$. Ce type de mise en scène se retrouve dans le texte de Luo Ying : ici aussi, les cadavres forment une exposition et les combats se confondent avec des spectacles. Mais, tandis que chez Lu Xun, l'attitude du public est

84. Ibid. : 48 .

85. Ibid.: 39 .

86. Ibid. : 64 .

87. Voir « Le garde rouge tombé sous le couperet » (ibid. : 79).

88. Veg $2009: 284-285$. 
comprise comme le résultat de la passivité d'un peuple chinois anesthésié par des siècles d'oppression impériale, dans le contexte de la Révolution culturelle, l'exhibition se mue en ostentation idéologique, en discours politique.

Dans la partie intitulée « Nous étions tous des gardes rouges », le poète met en scène les prolongements de la Révolution culturelle. La délation, l'esprit « voyou » et le style « caïd » sont autant de marques d'une lutte haineuse qui rappellent le comportement des gardes rouges ${ }^{89}$. Le langage continue, encore aujourd'hui, à véhiculer les topiques violentes de l'ère révolutionnaire : écrasé, brisé, envahi par les insultes, les malédictions, il est instrument d'agression. À cet égard, il est intéressant d'analyser la dialectique sous-jacente aux slogans hérités de la dernière période maoïste. Ces formules reposent sur des oppositions pronominales, dans lesquelles les deux termes prennent leur plein sens d'embrayeurs : «Toi, meurs, vive moi !» (Ni si wo huo), «Qui a peur de qui ?» (Shui pa shui ?), « Pour ceux qui sont avec moi, hourrah! Pour ceux qui sont contre moi, à bas!» (Shunwozhe chang, niwozhe wang). Les personnels et des interrogatifs, « moi », « toi », « qui », la violence de la sanction (vie, mort, peur) ramènent la personne humaine à un essentialisme sommaire, dans lequel le «toi » et le «moi » sont pareillement anonymes et interchangeables, se renvoyant la position de la jouissance cruelle et celle de la souffrance.

\section{En guise de conclusion : incorporation et vie contemporaine}

Victime de la violence politique, l'écrivain né dans les années difficiles de l'après-guerre a historiquement inscrit son écriture dans la description d'un danger vital : on ne peut manquer d'être frappé, en littérature chinoise contemporaine, par la représentation envahissante d'un être humain ramené à sa « somaticité ». Il n'est pas moins notable que cette thématique, loin de s'éteindre avec le souvenir des années de plomb, garde une présence inquiétante dans la littérature des dernières décennies. Cela pourrait être illustré chez des auteurs comme Mo Yan ou Yu Hua ; nous choisirons ici d'en donner un exemple dans «l'écriture féminine » (nüxing xiezuo). L'ensemble d'œuvres qu'on classifie sous cette appellation est marqué par l'influence des mouvements féministes et des théories littéraires venues d'Occident ${ }^{90}$; d'un texte à l'autre, on retrouve une dénonciation du corps politisé des héroïnes révolutionnaires et une quête de construction de l'identité féminine.

89. Luo Ying 2015 : 197.

90. À titre d'exemple, voir Zhang 1992 : on y trouve des articles de Simone de Beauvoir, d'Hélène Cixous, de Julia Kristeva, de Luce Irigaray. 
Au milieu des années 1990, un groupe de jeunes auteures s'impose sur la scène littéraire en publiant des textes à coloration autobiographique, caractérisés par une écriture du privé qui n'hésite pas à évoquer le désir physique des femmes, leurs fantasmes, les actes hétérosexuels, homosexuels ou les pratiques autoérotiques qui marquent leur sexualité ${ }^{11}$. La majorité de ces récits met en scène, à la première personne, un personnage féminin fuyant le monde extérieur ou en rupture avec la société. Cet isolement social semble déboucher sur l'affirmation d'un corps de type nouveau, pourvu d'une sexualité vécue de manière libre. On peut citer trois noms majeurs de ce mouvement : Hai Nan (1962-), Lin Bai (1958-) et Chen Ran (1962-) et des ouvrages représentatifs : Mes amants (Wo de qingrenmen), Le Combat solitaire (Yigeren de zhanzheng) et Ma vie privée (Siren shenghuo). Une autre écrivaine, Tie Ning (1957-), donne à l'un des ses romans un titre significatif : Les Grandes Baigneuses (Dayunii) ${ }^{92}$. Elle y met en scène une éditrice quadragénaire pour qui les anciennes valeurs féminines ne représentent que déception et qui voit dans la mondialisation l'occasion d'apprendre, découvrir de nouvelles valeurs et se reconstruire ${ }^{93}$. La délivrance du passé s'accomplit finalement au moment où l'héroïne découvre son propre corps - féminin, nu, dans sa maturité sexuelle ${ }^{94}$.

Mais le corps féminin, tel qu'il apparaît dans ces écritures, est bien plus souvent décrit comme une source de souffrance que d'épanouissement ${ }^{95}$. C'est particulièrement vrai dans les textes plus récents, de la génération des écrivaines nées dans les années 1970 (qui n’ont donc pas souvenir de la Révolution culturelle). Les récits de Wei Hui (1973-), auteure de Shanghai baby, ou de Mian Mian (1970-), auteure de Panda Sex et Les Bonbons chinois ${ }^{96}$, sont caractérisés par la mise en scène d'une marginalité néocapitaliste clinquante dans laquelle l'abondance matérielle va de pair avec la liberté sexuelle.

91. On a parlé de «Littérature de l'intimité féminine» (Nüxing yinsi wenxue).

92. Voir Tie Ning 2008. Le titre fait écho au tableau de Cézanne Les Grandes Baigneuses (1906) qui peint des nus féminins dans un paysage. Contrairement au tableau de Courbet Les Baigneuses (1853), celui de Cézanne fait naître, selon Philippe Sollers, une « inquiétude bisexuelle, qui rappelle l'histoire de L'Hermaphrodite endormi » (Sollers 1995 : 67-80).

93. Xu 2011a.

94. Tie Ning 2008 : 225, 226. Dans la scène du bain (la seule du roman), la jeune femme prend conscience de son corps au toucher de ses seins, de son sexe (225). La fusion corporelle avec Chen Zai, qui succède immédiatement à cette scène, représente le corps nu dans un plein épanouissement (225-226). La sexualité retrouve ainsi un aspect « naturel », dépouillé de toute connotation morale ou politique.

95. Sur les études à ce sujet, voir Zhang 2003, Jin 2002, Jin 2009.

96. Wei Hui 2001 ; Mian Mian 2001, Mian Mian 2009. 
Mais l'isolement des personnages, compris comme source de confort ou de révélation de soi dans la génération immédiatement antérieure, prend ici la coloration sombre d'une souffrance vécue sur fond de dépression et de drogue. Le texte devient le témoin de la vie d'un corps en proie à d'incontrôlables addictions, à une sexualité qu'il ne domine pas ou à des démons intérieurs qui prennent le visage de la folie. Nous retrouvons ainsi une à une les figures du corps diminué, de l'incapacité à régler une vie sexuelle (cette fois sur le versant féminin de l'excès, et non dans le registre masculin du défaut), de l'invasion délirante. Dans le roman de Wei Hui La Chambre de l'amant ${ }^{97}$, la protagoniste est une jeune femme sortie d'une hospitalisation en psychiatrie. Riche, elle se retranche dans un grand appartement de Shanghai dans lequel ses seuls contacts avec l'extérieur sont ceux que lui offre le voyeurisme. Satisfaire les désirs physiques d'un corps envahissant semble être pour elle le seul moyen de chasser le sentiment de vide et l'angoisse existentiels.

Cette invasion morbide par le corps se dit au pied de la lettre dans le roman de Sheng Keyi (1973-) Filles du Nord (Beimei) ${ }^{98}$. Au centre du récit, la particularité physique d'une jeune travailleuse migrante, Qian Xiaohong : d'énormes seins. Le personnage, brutalement sexué par cette caractéristique, voit sa vie s'organiser autour de celle-ci. Dans la lutte pour l'emploi qui régit l'existence des mingong, la poitrine de la jeune femme est un atout qui s'avère vite décisif : le roman dévoile «le corps féminin comme ultime atout dans la course à la réussite économique et à l'ascension sociale, avec les dérives liées $^{99} »$. Cette individuation ironique amène son cortège de mécomptes, physiques (avortements, maladie) et sociaux, des soucis de tous ordres venant assaillir la jeune femme. À la fin du roman, la poitrine de Qian Xiaohong, affectée d'une prolifération de glandes mammaires, devient extrêmement lourde. La scène finale met en scène une conclusion fantastique qui n'est pas sans rappeler, par-delà l'influence du réalisme magique marquezien, les descriptions délirantes de Zong Pu :

97. Wei Hui 2004.

98. Sheng 2011, Sheng 2012.

99. Voir la présentation du roman par Brigitte Duzan, http ://www.chinese-shortstories. com/Auteurs_de_a_z_Sheng_Keyi.htm, et celle d'Eric Abrahamsen pour sa traduction en anglais : http://paper-republic.org/ericabrahamsen/northern-girls-extract/ : « After arriving in Shenzhen Hong and her friend drift at the edges of society, working in hair salons, shops, factories and hotels, owning absolutely nothing in the world but their labor and their bodies.» 
Le corps souffrant dans la littérature chinoise depuis la Nouvelle période

Tombée par terre, Qian Xiaohong écrase ses seins [...]. Ces derniers semblent cloués dans le sol en ciment, Qian Xiaohong n'arrive pas à les tirer [...]. Elle serre les dents, baisse la tête, traîne ses deux seins aussi lourds que deux sacs de sable, sort de l'encerclement des pieds de la foule, descend le pont, pénètre dans les rues en rampant ${ }^{100}$.

La particularité physique représente ici symboliquement un poids insupportable. La vie sexuelle sans attaches de Qian Xiaohong apparaît ainsi plus comme une aliénation que comme une libération : dégagé de la collectivité initiale, le personnage ne peut plus retrouver un espace commun. L'individuation par le fait du corps se révèle fatale : dans la mesure où elle détache le sujet de la masse, elle le condamne à l'isolement et à la perte du repère du réel.

Le modèle de cette singularisation par le corps est certes très différent de celui qu'on voit mis en scène dans les textes issus de l'expérience de la Révolution culturelle. Le rapprochement n'en demeure pas moins clair. Dans une société néocapitaliste dans laquelle le corps se singularise par la multiplication des repères sociaux, du vêtement jusqu' au moyen de transport ou à la chirurgie plastique, la singularité se met en scène dans l'ordre du stigmate, du danger social et psychologique. Le corps ne survit qu'en s'individuant et, en s'individuant, se fait souffrant : l'équation est récurrente. Le paradoxe, qu'on voit se faire jour dans les tourmentes de la Révolution culturelle, se révèle ainsi à l'issue de l'analyse un phénomène moral complexe qui dépasse de beaucoup l'événement historique. Entre l'influence d'un puritanisme traditionnel aux couleurs bouddhistes ou maoïstes et la difficulté confucéenne à penser l'homme en dehors de la collectivité dans laquelle il s'inscrit, le corps se trouve à la croisée des inquiétudes. Ainsi, son image littéraire est ballottée entre difformité et violence : puni pour la manière dont il s'extrait d'un corps collectif dont la disparition progressive laisse un vide perçu comme effrayant, puni pour avoir usé de la liberté dont semblait le pourvoir la société nouvelle, le corps se représente - se vit, peut-être - comme un malade social.

Les derniers avatars de cette difficile appréhension sont particulièrement intéressants dans la mesure où ils semblent mettre en scène de nouveaux développements, plus radicaux encore. Selon Statistical Report on Internet Development in China, parmi les nombreux genres littéraires étiquetés sur les sites internet, une quantité très importante représente l'aspiration du corps souffrant à un au-delà, historique, transcendant, etc. : romans de cape et d'épée du monde des immortels (Xianxia), récits fantastiques (Xuanhuan), romans de

100. Sheng $2011: 279$. 
science-fiction (Chuanyuan) ${ }^{101}$. Comment le corps est-il représenté dans cet espace virtuel avec les outils des nouveaux médias ? Dans quelle mesure est-il encore corps physique ? Comment les éléments de sa surhumanité répondentils à la problématique du corps souffrant ? Il sera intéressant d'en proposer une nouvelle étude et de s'aventurer entre monde réel et monde virtuel ${ }^{102}$.

101. Voir Chen \& Song 2010. Selon une statistique de 2007, dans le palmarès des romans les plus lus, 249 récits parmi les 400 premiers classés mettent en scène un espacetemps différent du nôtre, soit une proportion de $62 \%$. (Pour indication : selon les statistiques publiées par « The China Internet Network Information Center » (CNNIC) indiquent environ $40 \%$ des internautes en Chine ont accès à la littérature en ligne. Hockx 2015 : 5.)

102. Voici nos premières études sur la web-littérature liées à ce sujet : sur le corps souffrant et l'écriture de la fantasmagorie, voir Xu 2013b ; sur l'imaginaire d'un corps utopique, voir Xu 2015. 
Le corps souffrant dans la littérature chinoise depuis la Nouvelle période

\section{BIBLIOGRAPHIE}

BEI DAO 北島 (1986), Guilaide moshengren 歸來的陌生人 (Un étranger de retour), Guangzhou, Huacheng chubanshe.

BEI DAO 北島 (1999). «Un étranger de retour ».In 13, rue du bonheur. Trad. CHENANDRO, Chantal. Paris, Circé.

CHEN, Qijia 陳奇佳, SONG, Hui 宋暉 (2010). «Wangluo xiaoshuo yu chaoyuexing yuansu 網絡小說與超越性元素 》(Romans d'internet et aspirations vers l'audelà). In Bei weiguande shizijia : jidujiao wenhua yu zhongguo dangdai dazhong wenxue 被圍觀的十字架: 基督教文化與中國當代大衆文學. Pékin, Zhongguo shehui kexue chubanshe : 487- 491 .

ClercQ, Martine (de) (2015). «Confessions d'un garde rouge ou comment l'histoire dévore ses enfants ». In LuO, Ying, Le Gène du garde rouge. Trad. Xu, Shuang et CLERCQ (de), Martine. Paris, Gallimard : 227-228.

DAI, Jinhua 戴錦華 (1996). 《Nüxing wenxue he gerenhua xiezuo 女性文學和個人 化寫作 » (La littérature féminine et l'écriture personnalisante). Dajia 大家, $\mathrm{n}^{\circ} 1$.

DEGUY, Michel (1999). «La poésie fait mal ? ». Le Nouveau Recueil, $\mathrm{n}^{\circ} 52$ : 50-57.

DING, Ling 丁玲 (1952). Taiyang zhao zai Sangganhe shang 太陽照在桑乾河上(Le soleil brille sur le fleuve Sanggan). Pékin, Renmin wenxue chubanshe.

Dutrait, Noël (2006). " "Cicatrices", reportages et réflexion ». Petit précis à l'usage de l'amateur de littérature chinoise contemporaine. Arles, Philippe Picquier.

FoucAult, Michel (2011). «Droit de mort et pouvoir sur la vie ». Histoire de la sexualité, I. La volonté de savoir. Paris, Gallimard [1 $1^{\text {re }}$ édition 1975, réédition collection « Tel», 1994].

FOUCAULT, Michel (2014). Surveiller et punir. Paris, Gallimard [1 $1^{\text {re }}$ édition 1975, rééd. « Tel » 1993].

Foucault, Michel (dir.), Grange, J. (2003). Le Pouvoir psychiatrique. Cours au Collège de France. 1973-1974. Paris, Seuil/Gallimard.

HAN, Shaogong 韓少功 (2000), Papapa 爸爸爸. Trad. DUTRAIT, Noël. La Tour d'Aigues, L'Aube poche.

HARCOURT, Bernard (dir.) (2013). La Société punitive. Paris, Seuil/Gallimard.

HockX, Michel (2015). Internet Literature in China. New York, Columbia University Press.

JEANCOURT GALignani, Oriane (2013). « Luo Ying : l'enfer made in China ». Transfuge, $\mathrm{n}^{\circ} 70: 80$.

JIN, Siyan (2009). «Écriture du corps et mémoire intérieure : le je dédoublé ». In L'Écriture féminine chinoise du XX $X^{e}$ siècle à nos jours. Paris, Youfeng : 240-271.

JiN, Siyan (novembre-décembre 2002). « La littérature féminine dans la Chine d'aujourd'hui ». Perspectives chinoises, $\mathrm{n}^{\circ} 74: 44-54$.

LAmotTe, E. (trad.) (1944, 1949, 1970, 1976, 1980), Le Traité de la grande vertu de sagesse de Nāgārjuna (Mahāprajñāpāramitāsaāstra). Bureaux du Muséon, Louvain, vol. 1, 1944 ; vol. 2, 1949 ; vol. 3, 1970 ; vol. 4, 1976 ; vol. 5, 1980. 
LI, Lei 李鐳 (12-06-2009). «Lei Feng: Wo yuan yongyuan zuo yige luosiding 雷鋒 : 我願永遠做一個螺絲釘》. Renmin zhengxie bao 人民政協報. http://cpc.people. com.cn/GB/64093/64104/158494/9520860.html, page consultée le 15-05-2015.

LI, Zhiqiang 李志強 (28-9-2014). «Zhang Xianliang : bei hua youpai laogai 22 nian, diyige tupo xingjinqu zuojia 張賢亮: 被劃右派勞改22年, 第一個突破性 禁區作家》(Zhang Xianliang : ancien droitier ayant passé 22 ans en camp de rééducation, premier écrivain à avoir transgressé le tabou de la représentation sexuelle). Xinjingbao 新京報. http://news.xinhuanet.com/politics/201409/28/c_127042679.htm, consultation du 19 mai 2015.

LUO YING 駱英 (2011). Di jiu ye 第九夜 (La neuvième nuit). Taibei, Eryu chubanshe.

LUO YING 駱英 (2013). Lümudu 綠母度 (Tara). Pékin, Renmin wenxue chubanshe.

LUO YING 駱英 (2013). Lapins, lapins. Trad. XU, Shuang, CHARDOUX Martine. Pantin, Le Castor Astral.

LUO YING 駱英 (2014). Wenge jiyi 文革記憶(Le Gène du garde rouge). Taibei, Eryu wenhua.

LUO YING 駱英 (2015). Le Gène du garde rouge (Wenge jiyi). Trad. Xu, Shuang, CLERCQ, Martine (de). Paris, Gallimard.

MAO, Zedong 毛澤東 (1963), «Interventions aux causerie sur la littérature et l'art à Yan'an ». In CEuvres choisies. Pékin, Éditions en langues étrangères, t. 3 : 67-99.

MAO, Zedong 毛澤東 (1993), «Wenyi gongzuozhe yao tong gongnongbing jiehe 文藝 工作者要同工農兵結合》 (Le travailleur de la littérature et de l'art doit s'associer aux ouvriers-paysans-soldats : discours prononcé le 28 mai 2015 aux causeries de Yan’an). Mao Zedong wenji 毛澤東文集 (Euvres de Mao Zedong), t. 2. Pékin, Renmin wenxue chubanshe.

MIAN MIAN 棉棉 (2000). Zai qidai zhong在期待中 (Dans l'attente). http ://edu.sina. com.cn/writer/mm/62.html, page consultée le 15-05- 2015.

MiAn Mian 棉棉 (2001). Les Bonbons chinois. Trad. GENTIL, Sylvie. Paris, Éditions de l'Olivier.

Mian Mian 棉棉 (2009). Panda Sex. Trad. Gentil, Sylvie. Vauvert, Au Diable Vauvert. Oliveira Gomes (de), Ariadna (2012), « L'exil hors du langage ». In CHEN-ANDro, Chantal, SAKAÏ, Cécile, XU, Shuang (dir.), Imaginaires de l'exil dans les littératures contemporaines de Chine et du Japon. Arles, Philippe Piquier : 185201.

RABUT, Isabelle (2005). « Yu Hua et l'espace hanté ». Les Temps modernes, $\mathrm{n}^{\circ}$ 630$631: 212-246$.

RoBERTS, Rosemary (2009). « Maoist Women Warriors : Historical Continuities and Cultural Transgressions ». In TAO, Dongfeng et al. (dir.), Chinese Revolution and Chinese Literature. Londres, Cambridge Scholars Publishing : 139-160.

SHENG, Keyi 盛可以 (2011). Beimei 北妹 (Filles du nord). Tianjin, Tianjin renmin chubanshe. $1^{\text {re }}$ publication 2003 sous le titre de Huoxiaqu 活下去 (Survivre) dans la revue Zhongshan.

SHENG, Keyi 盛可以 (2012). Northern Girls (Beimei). Trad. Shelly BRYANT. Melbourne, Penguin Books Australia.

Sollers, Philippe (1995). Le Paradis de Cézanne. Paris, Gallimard. 
Le corps souffrant dans la littérature chinoise depuis la Nouvelle période

TAO, Dongfeng, YAnG, Xiaobin, RoberTs, Rosemary et YANG, Ling (dir.) (2009).

Chinese Revolution and Chinese Literature. Londres, Cambridge Scholars Publishing.

TIE NING 鐵凝 (2008). Dayunü 大浴女 (Les Grandes Baigneuses). Changsha, Hunan wenyi chubanshe.

VEG, Sébastien (2009), « Politique et spectacle ». Fictions du pouvoir chinois. Paris, Éditions des hautes études en sciences sociales : 283-288.

WEI HUI 衛慧 (2004). «La chambre de l'amant ». In CHEN Feng (dir.), Shanghai, fantômes sans concession. Paris, Autrement, « Romans d'une ville» : 5-26.

WEI HUI 衛慧 (2001). Shanghai baby. Trad. WHIST, Cora. Arles, Philippe Piquier.

XU, Shuang (2010). «Le futur dans le passé : l'intention temporelle dans Le Vieux Bateau de Zhang Wei ». In Duanmu, Mei et Tertrais, Hugues (dir.), Temps croisés I. Paris, La Maison des Sciences de l'Homme : 69-76.

XU, Shuang (2011a). "Construire une nouvelle identité féminine en Chine postrévolutionnaire : étude du roman Les Grandes Baigneuses de Tie Ning (1957-) ». In $4^{e}$ Congrès du Réseau Asie et Pacifique : Femmes, Écritures, Mondialisation dans l'Asie contemporaine. Paris, publication en ligne, site du Réseau Asie et Pacifique. http://www.reseau-asie.com/userfiles/file/G01_Xu\%20 Shuang.pdf. Page consultée le 19 mai 2015.

XU, Shuang (2011b). « Identités sociales en mutation. Étude des personnages romanesques dans la littérature urbaine en Chine post-révolutionnaire ». In DANIEL, Yvan et RaIBAud, Martine (dir.), Identités, images, représentation(s). Paris, Les Indes Savantes, à paraître.

XU, Shuang (2012). "Sea in Guchuan (The ancient Ship) : a study of the novel of Zhang Wei (1956-)». Revista de Estudos Chineses, $\mathrm{n}^{\circ} 8$ : 155-170.

XU, Shuang (2013a). « Figures du fantôme dans le roman sur Internet : L'Impératrice Phénix (Di Huang) de Tianxia Guiyuan ». In DURAND-DASTÈS, Vincent et LAUREILlARD, Marie (dir.), Une esthétique de la fantasmagorie : fantômes dans l'Extrême-Orient d'hier et d'aujourd'hui. Berne, Peter Lang, à paraître à l'automne 2015.

XU, Shuang (2013b). « L'écriture du fantastique chez Mo Yan ». In ZHANG, Yinde, XU, Shuang et DUTRAIT, Noël (dir.), Mo Yan, au croisement du local et de l'universel. Paris, Seuil : à paraître en 2016.

XU, Shuang (2014). "L'écriture du corps imaginaire comme expérience de la transgression ». In ZHANG, Yinde, Xu, Shuang et Dutrait, Noël (dir.), Mo Yan, au croisement du local et de l'universel. Paris, Seuil, à paraître en 2016.

XU, Shuang (2015). « Passing through time and space, looking for the utopia in web "chuanyue" novels ». In ZHANG, Yinde et WANG, David Der-Wei (dir.), Utopia and Utopianism in the Contemporary Chinese Context : Texts, Ideas, Social Spaces. Hong Kong, Hong Kong University Press, à paraître.

ZHANG, Jingyuan 張京媛 (1992), Dangdai nüxingzhuyi wenxue piping 當代女性主 義文學批評 (Études de la littérature féministe contemporaine). Pékin, Beijing daxue chubanshe. 
ZHANG, Wei 張煒 (2014). Le Vieux Bateau (Gu chuan). Trad. BERGERET CURIEN, Annie et XU Shuang. Paris, Seuil.

ZHANG, Xianliang 張賢亮 (2009). Nanren de yiban shi nüren 男人的一半是女人

(La moitié d'homme, c'est la femme). Pékin, Zhongguo wenlian chubanshe ( $1^{\mathrm{re}}$ publication 1985, Shouhuo, $\left.\mathrm{n}^{\circ} 5\right)$.

ZHANG, Xianliang 張賢亮 (2004). La moitié de l'homme, c'est la femme. Trad. YANG, Yuanliang et LOI, Michelle. Paris, Belfond.

ZHANG, Yinde (2003). «Du shishôsetsu à l'écriture féminine : l'exemple de Chen

Ran ». In ZHANG, Yinde, Le Monde romanesque chinois au XX siècle : modernité et identités. Paris, Honoré Champion : 393-415.

ZHANG, Yinde (2014). Mo Yan, le lieu de la fiction. Paris, Seuil.

Zhongguo wenxuejia cidian 中國文學家辭典 (Dictionnaire des littérateurs chinois) (1985), t. 4. Chengdu, Sichuan wenyi chubanshe.

Zong, $\mathrm{Pu}$ 宗璞 (1988) 《Ma coquille»(Woju 蝸居). La Remontée vers le jour, nouvelles de Chine(1978-1988). Trad. CHEN-ANDRO, Chantal. Aix-en-Provence, Alinéa : 25-35.

ZoNG, Pu 宗璞 (1994). «Qui suis-je ?»(Wo shi shui 我是誰). Trad. GIPOULON, Catherine. In CURIEN, Annie (dir.), Anthologie de nouvelles chinoises contemporaines. Paris, Gallimard : 31-38.

\section{GLOSSAIRE}

ban ge ren 半個人

Bei Dao 北島

Beimei 北妹

Chen Ran 陳染

chongzi 蟲子

Chuanyuan 穿越

da duchong 大毒蟲

Dayunü 大浴女

Di jiu ye 第九夜

Ding Ling 丁玲

duoluo 墮落

feiren 廢人

Guchuan 古船

Guilai de moshengren 歸來的陌生人

Han Nan 海男

Han Shaogong 韓少功

kang 康

laodongli 勞動力 
Le corps souffrant dans la littérature chinoise depuis la Nouvelle période

laogai 勞改

Lei Feng jingshen 雷鋒精神

Lin Bai 林白

Lu Xinhua 盧新華

Lu Xun 魯迅

$L \ddot{u m} u d u$ 綠母度

Luo Ying 駱英

Luosiding jingshen 螺絲釘精神

Mian Mian 棉棉

Minggong 民工

Mo Yan 莫言

Mozhang 魔障

Nanren de yiban shi nüren 男人的一半是女人

nei fenmi 內分泌

Ni si wo huo 你死我活

niugui sheshen 牛鬼蛇神

nuli 奴隸

nüxing xiezuo 女性寫作

Nüxing yinsi wenxue 女性隱私文學

Papapa 爸爸爸

qiutu 囚徒

renmin 人民

Shanghen wenxue 傷痕文學

Sheng Keyi 盛可以

Shiji 史記

shizhong 示眾

Shui pa shui 誰怕誰

Shunwozhe chang, niwozhe wang 順我者昌, 逆我者亡

silie 撕裂

sinanzhe 死難者

Siren shenghuo 私人生活

Tie Ning 鐵凝

tuotai huangu 脫胎換骨

Wei Hui 衛慧

Wenge jiyi 文革記憶

wenge jiyin 文革基因

Wo de qingrenmen 我的情人們

Wo shi shui 我是誰

xianjin 陷阱

Xianxia 仙俠 
Xu Shuang et Ariadna de Oliveira Gomes

xiaokang 小康

Xiao tuzi 小兔子

Xin shiqi 新時期

Xuanhuan 玄幺𠃌

Yigeren de zhanzheng 一個人的戰爭

yinxian 陰險

Yu Hua 余華

Zhang Wei 張煒

Zhang Xianliang 張賢亮

zhengzha 掙扎

Zong $\mathrm{Pu}$ 宗璞 